\title{
LA CIENCIA POLÍTICA EN BRASIL EN LA ÚLTIMA DÉCADA: LA NACIONALIZACIÓN Y LA LENTA SUPERACIÓN DEL PARROQUIALISMO*
}

\author{
Political Science in Brazil in the Last Decade: Nationalization and the \\ Slow Overcoming of Parochialism
}

\section{OCTAVIO AMORIM NETO}

Escuela Brasileña de Administración Pública y de Empresas (EBAPE)

Fundación Getúlio Vargas (FGV), Rio de Janeiro

\section{FABIANO SANTOS}

Instituto de Estudios Sociales y Políticos (IESP)

Universidad del Estado de Rio de Janeiro (UERJ)

\begin{abstract}
RESUMEN
El período 2005-2014 fue una de las mejores décadas de la Ciencia Política (CP) brasileña. Para que esto ocurriera, contribuyeron, en primer lugar, el crecimiento de la economía del país y el consecuente aumento del gasto público dedicado a las universidades y a la actividad científica en general y las políticas del Ministerio de Educación. También fue vital para este éxito la acción colectiva eficaz en el seno de la comunidad de politólogos brasileños. Consecuentemente, la CP brasileña se encuentra hoy, en el contexto latinoamericano, más cercana al potencial generado por la población y la economía del país. Además, la disciplina ha sido nacionalizada y su agenda de investigación hoy es menos parroquial. Por el lado negativo, sin embargo, se mantiene la relativamente baja visibilidad internacional de la producción de los politólogos brasileños, se constata la escasa aplicación fundamentada de métodos cualitativos y, recientemente, ha aflorado una considerable inseguridad sobre los recursos disponibles para la disciplina, teniendo en cuenta los años de ajuste fiscal que se avecinan.
\end{abstract}

Palabras clave: Ciencia política, Brasil, institucionalización tardía, baja visibilidad internacional, acción colectiva.

\begin{abstract}
The years between 2005 and 2014 were one of the best decades of political science (PS) in Brazil. The growth of the economy, the consequent increase in public spending on universities and scientific activity in general, and the policies pursued by the Ministry of Education played a key role in bringing this outcome about. Effective collective action within the Brazilian PS community was also a vital contributing factor. Consequently, Brazilian PS is now closer to the potential generated by the country's population and economy, as far as the Latin American context is concerned. In addition, the discipline has been nationalized, and its research agenda is now less parochial nowadays. On the negative side, however, the output of Brazilian political
\end{abstract}

* Los autores agradecen los comentarios del revisor anónimo y de Andrés Malamud y Diego Rossello a una primera versión de este artículo y la ayuda de Igor Acácio y Mariana Carvalho Barbosa en la investigación. Renata Oliveira Rufino hizo la traducción del portugués al español. 
scientists still stands out for its relatively low international visibility. Moreover, Brazilian PS remains characterized by a scarce systematic application of well thought-out qualitative methods. Finally, a considerable uncertainty over the resources available to the discipline has recently surfaced, given the years of fiscal adjustment lying ahead.

Key words: Political science, Brazil, late institutionalization, low international visibility, collective action.

En nuestro artículo sobre la ciencia política brasileña, publicado hace exactamente una década (Amorim Neto y Santos, 2005), sostenemos que esta logró el prodigio de establecerse en la década de 1970 bajo un régimen militar, llegando, en las décadas siguientes, a crecer e institucionalizarse como disciplina académica con la creación de nuevos programas de maestría y doctorado. Matizamos ese diagnóstico optimista al alertar sobre el hecho de que, a pesar de los avances, había aún importantes desafíos por delante, como la superación de grandes dificultades económicas y la escasa cohesión entre los pares.

En ese momento temíamos que, si la comunidad de politólogos fracasase en la solución de aquellos problemas, la ciencia política (de ahora en más $\mathrm{CP}$ ) brasileña correría riesgos en mediano plazo. Específicamente para la cuestión relativa a la escasa cohesión entre los pares, sugerimos un modelo consociacional, al estilo de Lijphart, de interacción inter e intradepartamentales. En las palabras que usamos hace 10 años: "Para esto, obviamente, hay que generar un amplio consenso en torno a las líneas de expansión de la comunidad. Este consenso todavía no se ha movilizado, pero, para nosotros, está claro que se debe fundar en el fortalecimiento de la enseñanza de métodos en los cursos de graduación y posgrado, así como en el perfeccionamiento de la enseñanza de humanidades en el curso de graduación" (Amorim Neto y Santos, 2005: 110).

¿Fue correcto el diagnóstico que hicimos en 2005? ¿Se adoptaron las soluciones que propusimos? ¿Cuáles fueron las políticas académicas que siguió la CP brasileña en los últimos 10 años? ¿Qué resultados se obtuvieron? ¿Cómo se encuentra hoy la disciplina?

Anticipando de manera muy breve nuestras respuestas, las dificultades que habíamos identificado fueron parcialmente superadas, pues, en los últimos años, se observaron, por el lado positivo, más coordinación, solidaridad y acción colectiva en el seno de la comunidad de politólogos brasileños, la consolidación del uso sistemático y sofisticado de los métodos estadísticos y más recursos para la investigación y para la creación y expansión de departamentos. Por el lado negativo, sin embargo, se mantiene la relativamente baja visibilidad internacional de la producción de los politólogos brasileños, se constata la escasa aplicación fundamentada de métodos cualitativos y, recientemente, ha aflorado una considerable inseguridad sobre los recursos disponibles para la disciplina, teniendo en cuenta los años de ajuste fiscal que se avecinan.

Dicho lo anterior, el artículo está estructurado de la siguiente manera. La sección siguiente reseña la reciente bibliografía sobre la evolución de la CP brasileña, ofreciendo datos primarios y secundarios que muestran, con cierto detalle, la expansión institucional observada en los últimos 10 años, la nacionalización de la disciplina, la ampliación del 
número de cursos de grado y posgrado, el aumento de la producción de artículos en revistas nacionales e internacionales, y la temática de las principales agendas de investigación. En la tercera sección, presentamos nuestro diagnóstico de las principales tendencias de los últimos diez años, en las que se destacan la lenta superación del parroquialismo por medio de la consolidación de la lógica interna de la agenda de investigaciones sobre el presidencialismo comparado y la parcial superación de las deficiencias metodológicas identificadas por Soares (2005). La última sección enuncia las principales conclusiones, especulando también sobre los desafíos y riesgos de la próxima década.

\section{QUÉ DICEN LOS POLITÓLOGOS SOBRE LA CIENCIA POLÍTICA BRASILEÑA}

En esta sección reseñamos tres artículos muy recientes sobre la CP brasileña: los dos primeros versan sobre la institucionalización y autonomización de la disciplina y el último sobre el contenido temático de la producción científica, además de dos artículos, también recientes, que permiten encuadrar el país en el contexto latinoamericano. Con base en los datos proporcionados por estos ricos trabajos empíricos y en datos que hemos recolectado, verificamos si -y en qué medida- el diagnóstico que hicimos en 2005 todavía tiene validez.

Comenzamos con el excelente texto de Marenco (2014), que analiza, de manera percuciente, la institucionalización de la CP brasileña. El autor define la institucionalización de la disciplina como un proceso resultante de dos factores: la expansión de los indicadores de profesionalización -que incluyen las bases materiales necesarias para la actividad académica- y la formación de los profesionales dedicados a la investigación en universidades y asociaciones profesionales; $y$ la consolidación de un sistema de evaluación institucional basado en el parecer de los pares que sirva para el establecimiento de jerarquías y la destinación de recursos e incentivos a la actividad académica.

Al considerar el segundo factor, Marenco identifica como el principal impulsor de la institucionalización de la CP en Brasil la creación del sistema de evaluación de los cursos de posgrado por el Ministerio de Educación en 1976, que tiene dos aspectos clave: la habilitación de nuevos cursos y la evaluación trienal del desempeño de todos los cursos de posgrado, con el otorgamiento de una nota para cada departamento.

$\mathrm{Al}$ abordar el primer factor, Marenco destaca la expansión de las instituciones académicas como la gran fuerza motriz de la profesionalización. En este sentido, la formación de grado o licenciatura en CP llegó tarde a las universidades brasileñas, si la comparamos con la Sociología y la Antropología (la primera carrera de grado en CP fue creada recién en 1996). Sin embargo, en la última década, se han producido grandes cambios con el aumento de la oferta de carreras de grado en relaciones internacionales (RI) y políticas públicas (PP), cursos que se combinan a los de CP. Hoy en día, en Río de Janeiro, por ejemplo, la Universidad Federal del Estado de Rio de Janeiro (UNIRIO) ofrece una carrera de grado en CP y RI, y lo mismo ocurre en Pernambuco, en la Universidad Federal de Pernambuco (UFPE), y en la también Universidad Federal de Piauí (UFPI), mientras que en Minas Gerais, en su Universidad Federal (UFMG), la formación en CP se da en 
el contexto de la carrera de gestión en PP, de la misma forma que en la Universidad Federal de Rio Grande do Norte (UFRGN). Respecto de los cursos de posgrado, la nacionalización también se ha observado. En 2004, solo seis estados brasileños contaban con programas de posgrado en ciencia política y relaciones internacionales. En 2011, ese número se elevó a 12 unidades de la federación (Madeira 2013), con especial mención para la creación de la maestría en Paraná (UFPR) y en Pará (UFPA) y del doctorado, también en Paraná, en la UFPR, y en la ciudad paulista de São Carlos (UFSCAR). El Gráfico 1, a continuación, muestra la evolución del número de carreras de grado y programas de maestría y doctorado en CP y RI entre 2005 y 2014. Cabe señalar que la tasa de incremento fue mayor que el $100 \%$ en los tres niveles.

\section{Gráfico 1. Número de Carreras de Grado (o Licenciatura) y Programas de Maestría y Doctorado en Ciencia Política y Relaciones Internacionales en Brasil en 2005 y 2014}

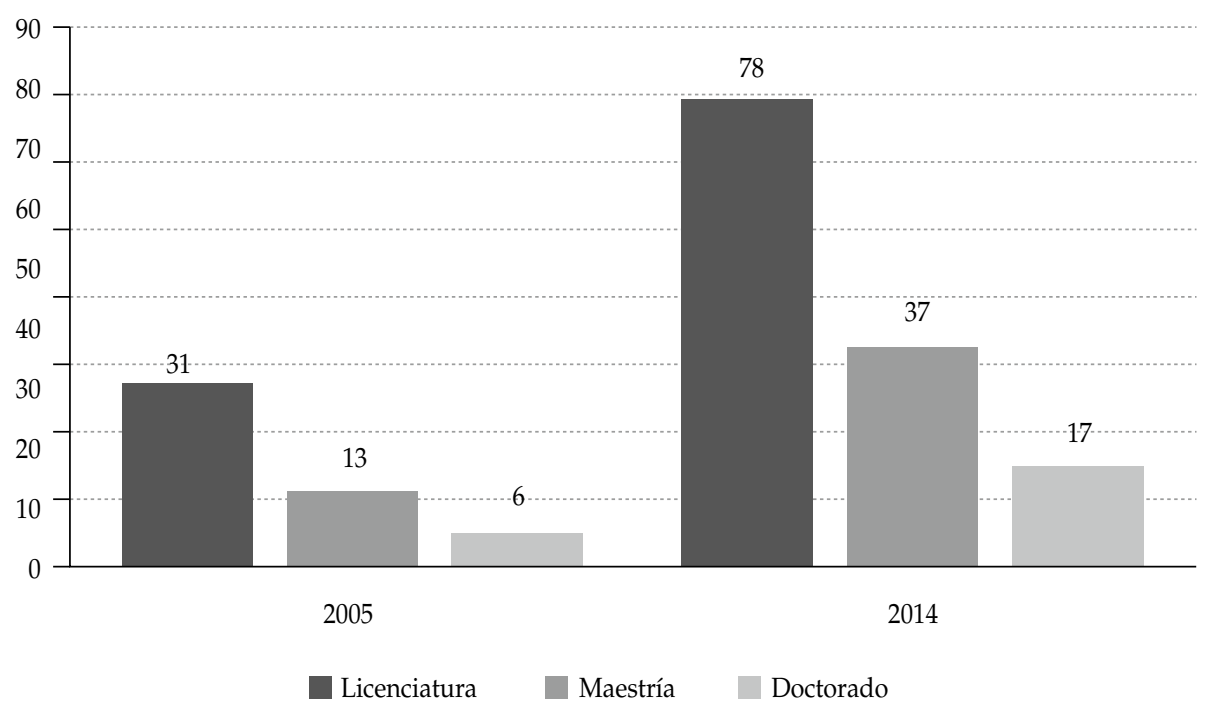

Fuentes: Para los datos sobre el posgrado, ver http:/ / conteudoweb.capes.gov.br, fecha de consulta: 24 de febrero de 2015. Para los datos sobre las carreras de grado, ver http:/ /emec.mec.gov.br/, fecha de consulta: el 27 de febrero de 2015.

Nota: Cuatro programas de maestría (UFBA, UNILA, EGN y UFABC) y el programa de doctorado de la UFPR todavía aguardaban la acreditación. El programa de la UFRJ existía en 2005, pero en 2008 el Ministerio de la Educación revocó su acreditación. También se incluyeron, para 2005, los programas de maestría y doctorado del IUPERJ, que perdieron la acreditación del Ministerio de la Educación en 2013. Cuanto a los datos del grado se excluyeron los conteos duplicados. Solo se consideraron carreras de la misma institución si presentaran ubicación y fecha de fundación diferentes. No está disponible la información sobre la fecha de fundación de cinco carreras de Relaciones Internacionales (UNICID, FADITU, UNIAN, FESBH y UFT). Se excluyeron doce cursos de Relaciones Internacionales porque no había información oficial disponible sobre las grillas curriculares, y se excluyeron otros 55 cursos de Relaciones Internacionales porque no presentaban contenido sustancial de Ciencia Política o se enfocaban en Comercio Exterior o Negocios Internacionales. Cuanto a los cursos dedicados a Políticas Públicas se incluyeron los de "políticas públicas" y de "gestión de políticas públicas". 
La dinámica de las carreras de posgrado, sin embargo, permite una medida más adecuada del comportamiento de las comunidades científicas. El posgrado en CP tuvo inicio en 1965 en Brasil, con la creación de la maestría en la Universidad Federal de Minas Gerais (UFMG), seguida, en 1969, por la maestría en CP en el antiguo Instituto Universitário de Pesquisas do Rio de Janeiro (IUPERJ), actual Instituto de Estudios Sociales y Políticos (IESP). El número de doctorados en CP, Sociología y Antropología fue similar hasta 1983, año a partir del cual hubo un aumento del número de cursos de Sociología. A partir de 2003, sin embargo, se inició una expansión de la CP, principalmente a nivel de maestría, como se puede verificar en el Gráfico 1.

La CP, no obstante, tiene una presencia aún menor que la Sociología y la Antropología en Brasil, lo que genera un déficit en la formación de doctores y en la creación de nuevas instituciones de posgrado en aquella disciplina. De forma complementaria, Marenco destaca otro efecto negativo de la institucionalización tardía de la CP brasileña. Recién en 2010, dos programas de CP -el de la Universidad de São Paulo (USP) y el de la IUPERJ / IESP- lograron obtener la nota máxima del Ministerio de Educación (las carreras de sociología y antropología ya la habían obtenido en 2001). ${ }^{1}$

Respecto de uno de los principales indicadores de productividad científica, el número de artículos publicados en revistas indexadas, Marenco (2014) muestra que la CP brasileña pasó de la $44^{\mathrm{a}}$ posición en 1998 a la $16^{\mathrm{a}}$ en 2012, probablemente debido a una mejora en la indexación de las revistas brasileñas y a la inclusión de estas en la base de datos Scopus. Desde 2004, Brasil se ha consolidado como líder en las publicaciones indexadas en América Latina, superando a Argentina, Chile y México. Aunque, si se consideran las menciones de las publicaciones de los politólogos brasileños, el comportamiento es mucho más irregular, y oscila entre la $68^{\text {a }}$ posición, en 2002, la $20^{\text {a }}$, en 2010 , y termina en la $38^{\circ}$, en 2012. Es decir, la CP brasileña está publicando más, pero su producción académica aún posee baja repercusión en la comunidad internacional. Marenco destaca el hecho de que parte significativa de la producción brasileña se publica en portugués, en revistas brasileñas, con escasa colaboración internacional, y aborda temas que son territorialmente limitados y se relacionan con la política brasileña. Además, el volumen de la producción brasileña en trabajos de teoría política (tanto normativa como positiva) o en estudios comparados es limitado, y eso también ayuda a explicar la repercusión todavía baja de la creciente producción científica de la CP brasileña.

En resumen, para Marenco, la CP brasileña logró institucionalizarse y nacionalizarse, pero tardíamente con relación a la Sociología y a la Antropología. Esta es una calificación importante al diagnóstico que hicimos en 2005. Debido a los problemas que señala Marenco, los departamentos y profesionales de la disciplina, si la comparamos, sobre todo, con la Sociología, aún son pocos. En 2013, mientras el número de programas de

1 Las evaluaciones más recientes del Ministerio de Educación (área de CP y RI) indican una transformación significativa. No solo ha crecido la oferta de cursos de CP y RI, sino que los cursos de políticas públicas, gestión y estudios estratégicos -todos ellos bajo la jurisdicción de CP y RI en el ámbito del Ministerio de Educación- crecen cuantitativa y cualitativamente. Este proceso puede implicar cambios en el tamaño relativo de las disciplinas "hermanas" dentro de las ciencias sociales (CP, Sociología, Antropología). 
doctorado en Sociología llegaba a 37, el de CP era de apenas 17; en 2012, mientras el número de doctores en Sociología dividido por el número de profesores permanentes de la disciplina era de 0,33, el de CP era de 0,21 (Marenco, 2014: 15, 18). Por último, la producción de la $\mathrm{CP}$ brasileña es creciente, pero todavía poco relevante internacionalmente.

Leite y Codato (2013), complementando el artículo de Marenco, discuten la autonomía de la $\mathrm{CP}$ brasileña. Los dos autores sostienen que uno de los principales factores que, en paralelo a la expansión y a la institucionalización, orientan y definen el proceso de autonomía de la CP brasileña es el Sistema Qualis, del Ministerio de Educación, creado para evaluar la producción académica de las disciplinas universitarias en Brasil en términos de la publicación de artículos en revistas periódicas especializadas. El Qualis es un conjunto de definiciones y normas de clasificación que determinan el valor de los elementos que componen la producción académica, como la calidad de las publicaciones periódicas y de los libros. Debido a que las publicaciones periódicas brasileñas pasaron a depender cada vez más de Qualis para sobrevivir en el mundo universitario, este se volvió el factor más relevante para determinar la jerarquía de la producción académica, siendo capaz de promover ciertos posicionamientos teórico-metodológicos en detrimento de otros. Para Leite y Codato, la CP se está autonomizando por medio del fortalecimiento de los abordajes "politicistas". Y aquí es donde Qualis cumpliría su papel crucial, pues serían estos los abordajes predominantes en las publicaciones periódicas mejor clasificadas.

Oliveira y Nicolau (2013), por su parte, se proponen a describir la trayectoria de la CP brasileña evaluando la producción de los politólogos desde el período de nacimiento de la disciplina académica en el país en la década de 1960. ¿Qué temas se estudiaron y qué métodos de recolección y análisis de datos se utilizaron? Estas son las principales cuestiones que los autores buscan tratar. Para responder a las preguntas, los investigadores analizan los artículos que los politólogos brasileños publican en cinco de las principales y más importantes revistas periódicas de ciencias sociales del país: Brazilian Political Science Review (BPSR), Dados, Novos Estudos, Opinião Pública y Revista Brasileira de Ciências Sociais (RBCS). El período analizado va desde 1966 a 2013. Se clasificaron 858 artículos de politólogos publicados en las cinco revistas seleccionadas, el 65\% publicados en los últimos 13 años. Hubo un crecimiento considerable en la producción académica, que consiste en la causa y consecuencia de la institucionalización de la CP como disciplina.

Oliveira y Nicolau también presentan un perfil de los politólogos brasileños: 68\% son hombres; $74 \%$ son profesores y $93 \%$ trabajan en instituciones brasileñas. Después, identifican las instituciones a las que pertenecen: seis de ellas concentran el $55 \%$ de los autores (USP, IUPERJ/IESP, UFMG, Universidad de Brasilia [UnB], Centro Brasileiro de Análise e Planejamento [CEBRAP] y Universidad de Campinas [UNICAMP]). Además de la concentración institucional, también hay concentración regional: cerca de tres cuartos de los autores de los trabajos pertenecen a instituciones localizadas en la región Sudeste.

Entre las áreas temáticas que movilizaron a los politólogos a lo largo de las cinco décadas se destacan "instituciones políticas", "elecciones y comportamiento político" y "teoría e historia del pensamiento político". Las dos primeras fueron fundamentales para el acentuado crecimiento verificado en el siglo XXI. Por otro lado, Oliveira y Nicolau registran 
una producción relativamente pequeña de artículos sobre relaciones internacionales y políticas públicas. ${ }^{2}$ Además, desde el punto de vista de los casos nacionales estudiados, la CP brasileña es marcadamente parroquial.

Otro aspecto que Oliveira y Nicolau evaluaron es el relativo a los métodos de recolección de datos y a los instrumentos con los cuales estos se analizan. En los años 1960 predominaba el análisis no empírico y los análisis histórico-descriptivos. Sin embargo, a partir de los años 1990, las investigaciones empíricas empiezan a predominar. Según estos autores, este cambio en las orientaciones metodológicas de los trabajos puede explicarse por medio del proceso de maduración de la disciplina: además de la elección de sus objetos de estudio, una disciplina consolidada se define también por la elección de los métodos de recolección y análisis de datos. Considerando el método de recolección de datos, Oliveira y Nicolau afirman que la CP recurre básicamente a las fuentes oficiales (documentos y datos) en la búsqueda de evidencias para sus explicaciones sobre el sistema político brasileño. Por último, los autores buscan saber qué instrumentos se usan en los análisis. Las estadísticas descriptivas simples, como el cálculo de frecuencias y porcentajes, son los más recurrentes en todo el período analizado. Sin embargo, puede percibirse que el uso de otros métodos estadísticos viene ganando espacio en la producción, sobre todo a partir de la década de 2000. El método que experimentó el mayor crecimiento fue el análisis de regresión. $\mathrm{O}$ sea, la CP brasileña siguió una trayectoria similar a la de los otros países, sobre todo de los Estados Unidos, donde la investigación empírica creció con el uso de métodos cuantitativos más avanzados.

En resumen, para Oliveira y Nicolau lo positivo en la última década fue el crecimiento acentuado de la publicación de artículos de CP en Brasil y un considerable progreso en la adopción de métodos estadísticos sofisticados. Lo negativo es que los artículos se concentran en pocas áreas temáticas, y su producción está muy concentrada por los politólogos que trabajan en instituciones localizadas en la región Sudeste del país. Es decir, se dio la nacionalización de los departamentos de $\mathrm{CP}$, aunque no necesariamente la nacionalización de la producción bibliográfica de la disciplina.

Pasamos ahora a dos conocidos artículos sobre la CP latinoamericana (Altman, 2006; Freidenberg y Malamud, 2013), los que nos ofrecen un marco internacional de la CP brasileña más preciso que los textos reseñados arriba. Compararemos a Brasil con otras dos grandes naciones latinoamericanas en términos de población (hasta 2005), Argentina y México (hoy, Colombia tiene una población mayor que Argentina).

En primer lugar, Altman (2006) muestra que el porcentaje de carreras de grado en $\mathrm{CP}$ en Brasil (12,6\%) -tomando como denominador el total latinoamericano hasta 2005era menor que el de Argentina (23,1\%) y México (30,1\%), a pesar de que la población brasileña, en aquel año, constituyera $34,6 \%$ de la población latinoamericana, y las de

2 El hecho de que Oliveira y Nicolau hayan encontrado pocos artículos de RI también tiene que ver con el hecho de que hayan excluido la Revista Brasileira de Política Internacional (RBPI) y la Contexto Internacional (CI), las dos principales revistas brasileñas de RI, de su muestra. Malamud y de Luca (2012) incluyen las dos revistas en su análisis de la producción bibliográfica brasileña, y constatan que la CP brasileña es menos parroquial que Oliveira y Nicolau sugieren. 
Argentina y México 7,5\% y 20\%, respectivamente. La situación brasileña era un poco mejor en lo referente al posgrado. En relación con los cursos de maestría, los porcentajes de los tres países eran los siguientes: Argentina: 27,8\%; Brasil: 13,4\%; y México: 9,3\%; cursos de doctorado: Argentina: 44,8\%; Brasil: 17,9\%; y México: 11,9\%.

Altman también estudió la producción de artículos publicados en revistas de $\mathrm{CP}$ indexadas entre 1975 y 2005. Los porcentajes de los tres países fueron los siguientes: Argentina: 11,8\%; Brasil: 25,5\%; y México: 34,0\%.

En conclusión, de acuerdo con Altman, el lugar de la CP brasileña en el contexto latinoamericano, hasta 2005, nunca estuvo a la altura del potencial generado por el tamaño de su población, sea en lo referente al número de carreras de grado y posgrado en $\mathrm{CP}$, sea en la producción de artículos. Así, puede decirse también que la institucionalización tardía de la CP brasileña, identificada por Marenco (2014), también tuvo un impacto negativo en el desempeño de la disciplina cuando se la compara con la de sus pares en la región.

Freidenberg y Malamud (2013), en su investigación original sobre los politólogos emigrados del Cono Sur (Argentina, Brasil, Chile y Uruguay), ofrecen una perspectiva distinta de la $\mathrm{CP}$ brasileña, sobre todo cuando se la compara con su gran rival Argentina, que permite calificar las verificaciones cuantitativas de Altman. Los dos autores constatan un mayor porcentaje de retorno de los politólogos brasileños que obtuvieron un doctorado fuera de su país de origen que el de sus pares argentinos. La principal razón de la diferencia se encuentra en las debilidades del desarrollo institucional y académico de la $\mathrm{CP}$ argentina cuando se la compara con la brasileña. Más específicamente, los brasileños vuelven a Brasil porque, a una tasa más alta que la de los argentinos, encuentran en su país buenas ofertas de trabajo en un creciente mercado académico, las que les propician el reconocimiento institucional y las condiciones económicas para acompañar la comunidad global de la disciplina (Freidenberg y Malamud, 2013: 11). Los autores concluyen en que la superioridad cuantitativa de la $\mathrm{CP}$ argentina que Altman evidencia no significa superioridad cualitativa. Mientras tanto, la mayor comunidad de politólogos argentinos emigrados acaba dándole una mayor visibilidad internacional a la CP argentina que a la brasileña, un caso típico de brain gain (Freidenberg y Malamud, 2013: 12).

Al fin y al cabo, a pesar de su institucionalización tardía y de los efectos que esto tuvo en el ámbito nacional y latinoamericano, el desempeño de la CP brasileña, en función de los avances cualitativos y cuantitativos que Marenco (2014), Leite y Codato (2013) y Oliveira y Nicolau (2013) identificaron en los últimos 10 años, es, hoy, más cercana al potencial generado por la población y economía del país. Mientras tanto, como muestran Marenco (2014) y Freidenberg y Malamud (2013), persiste el problema de la relativamente baja visibilidad internacional que tiene la producción de los politólogos brasileños.

\section{UN DIAGNÓSTICO DE LAS TENDENCIAS DE LOS ÚLTIMOS DIEZ AÑOS}

La sección anterior dejó en claro que los últimos 10 años fueron un período muy positivo para la CP brasileña. Separar las condiciones necesarias de aquellas que fueron suficientes es la llave para entender este avance. A lo largo de la década, el crecimiento de la economía 
del país, las amplias inversiones públicas en la expansión de las universidades y en investigación científica y los incentivos generados por las políticas del Ministerio de Educación ayudaron mucho a que la disciplina despegara. Diríamos que tales ingredientes forman las condiciones necesarias para la expansión. Las condiciones suficientes, a su vez, tienen relación con la coordinación, solidaridad y acción colectiva en el seno de la comunidad de politólogos brasileños. Discutimos la evolución de las condiciones suficientes con más detalle en los próximos párrafos.

En 2005 acechaban a la CP dos potenciales conflictos que, si hubieran sido mal administrados, podrían haber llevado a su estancamiento. El primero es el conflicto alrededor de las relaciones entre CP y RI. El origen de la cuestión reside en el hecho de que una parte significativa de los internacionalistas considera la RI como una disciplina aparte, distinta de la CP, con credenciales, por lo tanto, para volverse un área autónoma de evaluación en el ámbito del Ministerio de Educación. Tal visión generó algunos debates importantes en el contexto de la Asociación Brasileña de Ciencia Política $(\mathrm{ABCP})$, además de la fundación, en 2005, de la Asociación Brasileña de Relaciones Internacionales (ABRI). Aunque la creación de un área exclusiva de RI siga en la agenda de los internacionalistas y esté en el horizonte de decisiones relevantes de los comités pertinentes del Ministerio de Educación, el hecho es que liderazgos académicos importantes de RI vienen manteniendo su producción y militancia institucional en el ámbito de la $\mathrm{CP}$, controlando puestos importantes de decisión, tanto en la $\mathrm{ABCP}$, como en el propio Ministerio de Educación.

Otro conflicto potencial consistió en el surgimiento, a mediados de la década pasada, de una falsa dicotomía entre los "teóricos" de la política y los practicantes de una CP "empírica". En nuestro artículo de 2005 dijimos que tal dicotomía había generado un problema de falta de cohesión y solidaridad interna, potencialmente capaz de socavar la coordinación de la acción colectiva necesaria para enfrentar los desafíos de la expansión y nacionalización de la CP. Como buscamos mostrar en la sección anterior, los datos indican que la CP se expandió y, de alguna forma, se nacionalizó. Por lo tanto, es plausible sostener que la superación de divisiones artificiales -basadas más en diferencias de estilos intelectuales y objetos de investigación, propias de una disciplina marcada por la pluralidad, que en divergencias reales- es una de las principales causas de la expansión y nacionalización de la CP brasileña. Es plausible sostener, sobre todo, que, si tales conflictos potenciales no se hubiesen acomodado, la $\mathrm{CP}$ difícilmente habría aprovechado el boom económico de la última década de forma mínimamente efectiva y eficiente.

Además, es importante evaluar -en lo referente al proceso de acomodación de conflictos que se mencionó con anterioridad- el papel de la ABCP como foro de convivencia de las diferentes tendencias presentes en el interior de la CP. La ABCP, que se fundó a mediados de la década de 1980, se mantuvo inactiva por muchos años debido al poder de atracción que ejercía la Asociación Nacional de Posgrado e Investigación en Ciencias Sociales (ANPOCS) sobre el conjunto de los politólogos -una comunidad relativamente pequeña, como hemos visto anteriormente, si tomamos, sobre todo, la Sociología como parámetro de comparación. Sin embargo, a mediados de la década de 1990 se percibió 
la relevancia de una institución propia de la $\mathrm{CP}$ capaz de incorporar y proporcionar una audiencia para las nuevas generaciones de politólogos -que no encontraban en la ANPOCS oportunidad, o espacio, para la presentación y reflexión crítica de sus investigaciones-. La necesidad de "encajar" la producción de la CP en los temas debatidos en la ANPOCS por antropólogos y sociólogos, en un contexto en el que la redemocratización ya no unificaba los intereses de investigación de la comunidad más amplia de científicos sociales del país, terminó por restringir la capacidad de absorción de dicha asociación de las nuevas tendencias y preocupaciones de los politólogos.

La ABCP creció de forma sólida a partir de la primera década del siglo XXI, en gran parte debido al aumento del número de áreas temáticas (AT) alrededor de las que sus encuentros nacionales y congresos intermediarios se organizan. El crecimiento del número de AT, a su vez, ha permitido una absorción cada vez mayor de politólogos en los espacios de actuación académica e institucional de la disciplina. Este factor ha sido decisivo, en nuestra opinión, para la resolución del falso conflicto entre teóricos y empiristas de la $\mathrm{CP}$, una vez que teoría y empirismo pasaron a ser parte de un amplio y variado conjunto de foros de debate y discusión de temas tratados tradicionalmente por la disciplina. Además, la $\mathrm{ABCP}$ creció de forma coordinada con los representantes del área de CP y RI del Ministerio de Educación, en el sentido de su expansión y nacionalización. A partir de 2008, los encuentros nacionales se organizaron en centros fuera del eje Rio de Janeiro/São Paulo/Minas Gerais, estados en donde había nacido la CP brasileña. En 2010 se eligió a Recife, una ciudad del estado de Pernambuco, en el noreste del país, como sede del encuentro. En 2012 fue el turno del estado de Rio Grande do Sul, ubicado en el extremo sur del país, y en 2014, la capital federal, Brasilia, ubicada en el centrooeste de Brasil, fue también la capital de la CP y de la RI.

En síntesis, se puede decir que la $\mathrm{ABCP}$ cumplió un rol relevante en la acción colectiva de la comunidad de la $\mathrm{CP}$ dedicada a superar los desafíos de la expansión y nacionalización, al permitir, por un lado, la continua absorción de nuevos cuadros, practicantes de perspectivas teóricas y metodológicas diversas, y diluir, así, el falso conflicto entre teoría y empirismo y, por otro, al organizar los encuentros nacionales en lugares fuera del eje Rio de Janeiro/São Paulo/Minas Gerais, dando visibilidad a la CP en el país en su totalidad.

Pasamos ahora a evaluar algunas de las más relevantes tendencias sustantivas de la $\mathrm{CP}$ brasileña en los últimos 10 años.

Uno de los principales avances que observamos en la última década fue el inicio de la superación de la agenda parroquial y de la lógica externa que, hasta hace poco, habían caracterizado indeleblemente a la CP en el país (Amorim Neto, 2010; Castro Santos y Coutinho, 2002; Codato, 2013; Marenco, 2014; Nicolau y Oliveira, 2013). Parroquialismo significa introversión, es decir, estudiar casi exclusivamente la política brasileña. En las palabras de Marenco (2014: 34-35),

En las últimas dos décadas, la Ciencia Política brasileña ha hecho importantes avances en el conocimiento de la morfología del 'presidencialismo de coalición' brasileño, la disciplina de los partidos en Brasil, el federalismo y las relaciones 
intergubernamentales en Brasil, el comportamiento de los electores brasileños, y el crecimiento de los gastos sociales y políticas públicas en Brasil. Los números relativos a la producción científica publicada en revistas brasileñas muestran una vocación para el estudio de un caso nacional específico.

Respecto de la lógica externa, seguimos la definición dada por Morlino (1991: 354): "[...] consiste en volverse consciente de un urgente o importante problema político y proceder a analizarlo". Así, en virtud del parroquialismo, la CP brasileña ha tenido escasas preocupaciones nomológicas y metodológicas, poca relevancia internacional e interés por la política comparada (Marenco, 2014: 34-35). Ya por su lógica externa, la disciplina, en Brasil, termina siendo excesivamente dependiente de la coyuntura política y de los intereses de las agendas estatales de fomento científico. Además, no se logran obtener los beneficios que una lógica interna genera para la producción de conocimiento pionero y acumulativo. De nuevo en las palabras de Morlino (1991: 354),

La segunda lógica, interna, se superpone a la primera [la lógica externa], en la medida en que una serie de estudios emprendidos sobre el impulso de eventos o procesos políticos externos percibidos como relevantes genera, entonces, más estudios, réplicas y debates. Dentro de esta lógica interna, puede hacerse una nueva distinción entre estudios 'semilla' o 'seminales' y estudios de 'cierre'. Los estudios seminales son artículos o libros que abren un campo de investigación o marcan el comienzo de un período de creciente incidencia en un tema particular. Estos dan origen a otros estudios que, frecuentemente, les deben sus hipótesis e ideas. Ejemplos de tales estudios son los textos de Sartori sobre el Parlamento (1963) o sus trabajos sobre los partidos políticos (cf. 1976 y 1982).

En los últimos diez años hay claros indicios de que la CP brasileña comienza a superar su tradicional parroquialismo en función, sobre todo, de la consolidación de una sólida agenda de investigación sobre el presidencialismo comparado, fruto de la gran preocupación de los politólogos brasileños respecto del llamado presidencialismo de coalición, que caracteriza el actual régimen democrático brasileño. A su vez, el estudio del presidencialismo comparado, de a poco, viene generando una lógica interna en la CP brasileña, que tendría como trabajo seminal el artículo de Abranches (1988). Para evitar injusticias para con algunos colegas, no vamos a citar las referencias bibliográficas que sugieren el posible inicio del fin del parroquialismo y el posible inicio de una lógica interna en el área de política comparada en Brasil. La mejor fuente para tales referencias son los trabajos del politólogo americano Timothy Power (ver, entre otros, Power, 2010; Chaisty, Cheeseman y Power, 2014).

Otra tendencia que se consolidó en la última década, también sugestiva del inicio del fin del parroquialismo, habla de las comparaciones implícitas presentes en trabajos sobre la política brasileña, como busca mostrar Amorim Neto (2010). Aunque tengan como foco la política brasileña, muchos trabajos de la CP brasileña mantienen un intenso diálogo con las principales teorías sobre la organización política de los regímenes democráticos, buscando desafiar hipótesis influyentes y esquemas analíticos de comparatistas residentes 
en los EUA y en Europa. Es decir, el intenso diálogo con teorías comparativas es, de hecho, una característica de la ciencia política brasileña. Es decir, a Brasil se aplica perfectamente lo que dijo Sartori (1990: 413) sobre Italia: “En Italia, los comparatistas multinacionales, por así decir, no son legión; pero casi todos nuestros politólogos son 'implícitamente' comparatistas en el sentido de que sus parámetros son comparativos".

\section{CONCLUSIÓN}

El período 2005-2014 fue una de las mejores décadas de la CP brasileña. Para que esto ocurriera, contribuyeron, en primer lugar, el crecimiento de la economía del país y el consecuente aumento del gasto público dedicado a las universidades y a la actividad científica en general y las políticas del Ministerio de Educación. También fueron vitales para este éxito el espíritu de acuerdo entre las subdisciplinas y la acción colectiva eficaz en el interior de la ABCP. Consecuentemente, la CP brasileña se encuentra hoy, en el contexto latinoamericano, más cercana al potencial generado por la población y la economía del país.

En lo negativo, cabe enfatizar la relativamente baja visibilidad internacional de la producción de los politólogos brasileños. También vale destacar, en la actualidad, la excesiva valorización de los métodos cuantitativos (necesarios) y la virtual ausencia de la enseñanza de métodos cualitativos (notablemente, los métodos de análisis histórico propuestos por Mahoney y sus colaboradores) en los cursos de metodología y técnicas de investigación. Por otro lado, las dificultades en las carreras de grado siguen siendo enormes -se han abierto las carreras, pero faltan profesores para dar cuenta de las disciplinas que se van a ofrecer. Además, los números relativos a la evasión de los alumnos no son animadores. ${ }^{3}$ Por último, el aumento del número de doctores en $\mathrm{CP}$ recientemente formados de modo inevitable chocará con los peores años que experimentarán la economía y las finanzas públicas brasileñas en los próximos cinco años. Para que estos jóvenes doctores no vean frustrados sus planes de vida, va a ser fundamental que los actuales programas de posgrado de la disciplina pasen a desarrollarse aún mejor en las evaluaciones oficiales del Ministerio de Educación, de modo que tengan más capacidad de competencia por recursos públicos respecto de las otras ciencias sociales. En esta batalla, es esencial que los politólogos brasileños pasen a publicar más en inglés. No serán tareas fáciles, pero las condiciones para el éxito están dadas.

De acuerdo con los datos que nos envió por correo electrónico la profesora Cristiane Batista, de la UNIRIO, la tasa de evasión de los alumnos de la carrera de grado en CP y RI es aproximadamente de 35\%. En la UFPE, el promedio de la tasa entre 2009 y 2012 es de 29,5\%, según los datos que nos envió por correo electrónico el profesor Marcus Melo. 


\section{REFERENCIAS BIBLIOGRÁFICAS}

Abranches, Sérgio H. H. de. 1988. "Presidencialismo de Coalizão: O Dilema Institucional Brasileiro". Dados 31 (1): 5-38.

Altman, David. 2006. "From Fukuoka to Santiago: Institutionalization of Political Science in Latin America". PS: Political Science and Politics 39 (1): 196-203.

Amorim Neto, Octavio. 2010. "A Política Comparada no Brasil: A Política dos Outros". En Horizontes das Ciências Sociais no Brasil: Ciência Política, Renato Lessa (org.), 321-340. São Paulo: Anpocs/Editora Barcarolla/Discurso Editorial/ICH.

Amorim Neto, Octavio y Fabiano Santos. 2005. "La Ciencia Política en el Brasil: El Desafío de la Expansión”. Revista de Ciencia Política 25 (1): 101-110.

Castro Santos, Maria Helena de y Marcelo J. V. Coutinho. 2002. "Política Comparada: Estado das Artes e Perspectivas no Brasil". Boletim Informativo e Bibliográfico de Ciências Sociais 54: 5-44.

Chaisty, Paul, Nic Cheeseman y Timothy J. Power. 2014. "Rethinking the 'Presidentialism Debate': Conceptualizing Coalitional Politics in Cross-Regional Perspective". Democratization 21 (1): 72-94.

Freidenberg, Flavia y Andrés Malamud. 2013. "Politólogos on the Run: Contrasting Paths to Internationalization of Southern Cone Political Scientists". Latin American Politics and Society 55 (1): 1-21.

Leite, Fernando B. y Adriano Codato. 2013. "Automatização e Institucionalização da Ciência Política Brasileira: O Papel do Sistema Qualis-Capes". Revista de Discentes de Ciência Política da UFSCAR 1 (1): 1-21.

Madeira, Rafael M. 2013. "Quem Faz e Como se Faz Ciência Política no Brasil: Notas de Pesquisa”. Trabalho preparado para apresentação no VII Congresso Latino-Americano de Ciência Política (ALACIP), Bogotá.

Malamud, Andrés y Miguel de Luca. 2012. "An Old World Yet to Discover? European Studies in the Latin American Southern Cone". European Political Science 11 (3): 325-336.

Marenco, André. 2014. “The Three Achilles' Heels of Brazilian Political Science”. Brazilian Political Science Review 8 (3): 3-38.

Morlino, Leonardo. 1991. "Political Science in Italy: Tradition and Empiricism". European Journal of Political Research 20 (3-4): 341-358.

Oliveira, Lilian y Jairo Nicolau. 2013. “A Produção da Ciência Política Brasileira: Uma Análise dos Artigos Acadêmicos". Ponencia preparada para presentación en el $37^{\circ}$ Encuentro Anual de la Asociación Nacional de Posgrado e Investigación en Ciencias Sociales (ANPOCS), Águas de Lindóia.

Power, Timothy J. 2010. “Optimism, Pessimism, and Coalitional Presidentialism: Debating the Institutional Design of Brazilian Democracy". Bulletin of Latin American Research 29 (1) 8-33.

Sartori, Giovanni. 1990. "Comparazione e Metodo Comparato". Rivista Italiana di Scienza Politica 20 (3): 397-416.

Soares, Gláucio A. D. 2005. "O Calcanhar Metodológico da Ciência Política no Brasil”. Sociologia, Problemas e Práticas 48: 27-52.

Octavio Amorim Neto es profesor adjunto de la Escuela Brasileña de Administración Pública y de Empresas (EBAPE) en la Fundación Getulio Vargas (FGV), Rio de Janeiro, y investigador del Consejo Nacional de Desarrollo Científico y Tecnológico (CNPq), de Brasil.

E-mail: oamorim@fgv.br

Fabiano Santos es profesor investigador del Instituto de Estudios Sociales y Políticos (IESP) en la Universidad del Estado de Rio de Janeiro (UERJ), y investigador del Consejo Nacional de Desarrollo Científico y Tecnológico (CNPq), de Brasil. Fue presidente de la Asociación Brasileña de Ciencia Política entre 2008 y 2012.

E-mail: fsantos@iesp.uerj.br 
